\title{
881
}

\section{Sensitivity of Activated Human Lymphocytes to Cyclosporine and Its Metabolites}

\author{
A. Zeevi, R. Venkataramanan, G. Burckart, C.P. Wang, \\ N. Murase, D.H. Van Thiel, T.E. Starzl, L. Makowka, \\ and R.J. Duquesnoy
}

ABSTRACT: Alloreactive $T$ cells generated as clones from mixed lymphocyte cultures, or propagated from beart or liver transplant biopsies, were tested for secondary proliferation measured in the primed lymphocyte test in the presence of Cyclosporine A and meiabolites fractionated from buman bile. Significant differences were observed in Cyclosporine A sensitivity between various cell cultures ranging as high as 100-fold. The liver is the primary site of Cyclosporine A metabolism, which yields a number of hydroxylated and N-dimetbylated derivatives that are eventwally secreted into the bile. Bile was collected from adult liver transplans patients on Cyclosporine A therapy and following ext raction with diethyl ether, separated by bigh pressure liquid chromatography. Thirteen fractions were tested for their effect on lymphocyle proliferation in concanavalin $A$ activation, mixed lymphocyte cultures and primed lymphocyte test assays. The strongest immunosuppressive effect was found with fraction 8, which contained mesabolite $M 17$, which bas a single bydroxylation in position 1. Only three other fractions 9,10 , and 13, which contained metabolites $M 1$, $M 18$, and $M 21$, respectively, exhibited immunosuppressive activity, albeit much lower than that of Cyclosporine A. Differences in Cyclosporine $A$ sensitivity among alloreactive $T$ cells followed similar patterns with Cyclosporine $A$ metabolites. Thus, the assessment of the Cyclosporine $A$ effect must consider differences in drug sensitivity of lymphocyles involved in transplant intmunity and the generation of metabolites with immunosuppressive activity.

ABBREVIATIONS

CsA Cyclosporine A IL-2 interleukin-2

MLC mixed lymphocyte culture PLT primed lymphocyte test

HPLC high pressure liquid chromatography

$\begin{array}{llll}\text { PBL } & \text { peripheral blood lymphocytes } & \text { LB } & \text { liver biopsy } \\ \text { TCM } & \text { tissue culture medium } & \text { SD } & \text { standard deviation }\end{array}$

Con A concanavalin A

\section{INTRODUCTION}

Although cyclosporine $A(C s A)$ has a powerful immunosuppressive effect on allograft rejection, many transplant recipients experience rejection episodes that are often irreversible. Rejection is generally mediated by activated $T$ cells infiltrating the allograft. Recently developed methodologies of propagating activated

From the Dirision of Clinical Immunopathology. Clinical Pharmacokinetics Laboratory and Department of Surgery and Gassroenterology. Unitersity of Pislsburgh. Pillsburgh. Pennsyliania.

Address reprins reques/s so Adriana Zeevi, $P$ bD. Dirision of clinical Immunoparbology. University of Pitlsburgh. Children's Tolver. Room 5725. Pillsburgh, PA 152137-3417.

Receired August 12. 1987: accepred December 15. 1987. 


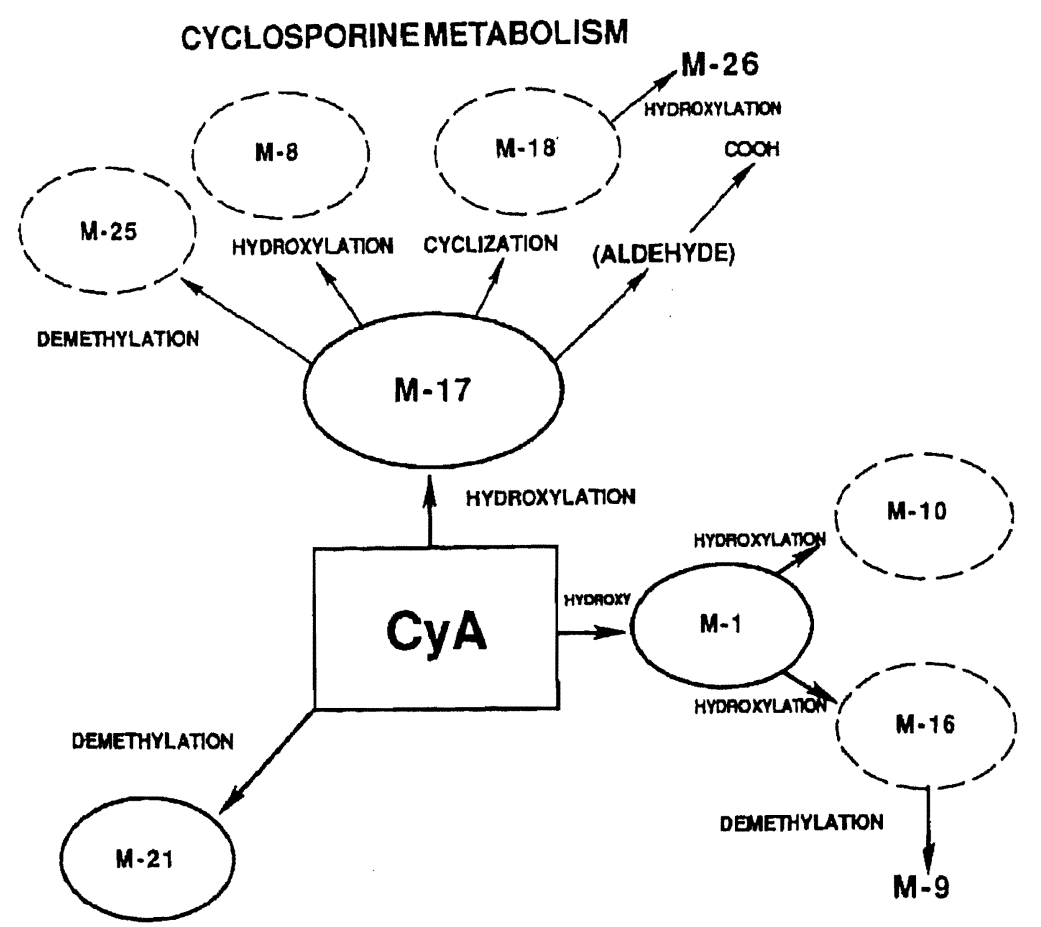

FIGURE 1 Schematical diagram of the pathways of Cyclosporine A metabolism.

$T$ cells from transplanted tissues have demonstrated the presence of donorspecific lymphocytes in transplanted organs at various stages of rejection [1-3]. Donor-specific $T$ cells have also been grown from transplant biopsies without any evidence of an ongoing or pending rejection episode as diagnosed clinically or histologically [4]. Therefore, the presence of donor-specific alloreactive $T$ lymphocytes in allografts may not be always associated with a rejection episode. Although absence of rejection might be explained by the presence of suppressor cells in the cellular infiltrates, it is also possible that differences in the sensitivity of graft-infiltrating lymphocytes to CsA may play a role. Several investigators have reported differences among individuals in CsA sensitivity of peripheral blood lymphocytes (PBL) activated by mitogens or in mixed lymphocyte culture (MLC) $[5,6]$.

From a practical standpoint, studies of CsA sensitivity of lymphocytes should also consider the metabolites of CsA. Structurally, CsA is a cyclic undecapeptide with several $N$-methylated amino acids and a unique unsaturated amino acid. Following administration, CsA is metabolized by hydroxylation and $N$-demechylation through the mono-oxygenase cytochrome $\mathrm{P} 450$ system primarily in the liver (Figure 1) [7]. The CsA metabolites are excreted into the bile and eventually eliminated through the intestinal tract [8].

Several CsA metabolites exhibit immunosuppressive activity as shown by in vitro assays with lymphocytes stimulated by mitogens such as phytohemagglutinin (PHA) and concanavalin A (Con A) or in mixed leukocyte reaction culture (MIR) [0,10]. Certain metabolites may also inhibit interleukin-2 (IL-2) production in MLC and the generation of cytotoxic T cells [10]. Particularly. metabolites M17 and M1 with single hydroxylations of amino acids in positions 1 
and 9, respectively, exhibit a considerable degree of immunosuppressive activity, which, depending on the type of in vitro assay used, approaches that of CsA [10]. On the other hand, metabolite M8, which is hydroxylated at both amino acids 1 and 9, as well as metabolite $M 21$, which is $N$-methylated at amino acid in position 4, exhibit markedly reduced in vitro immunosuppressive activity [10].

The chromatographic fractionation of CsA metabolites in human bile from liver transplant recipients has been previously reported [11]. Several fractions, obtained by high pressure liquid chromatography (HPLC) have now been tested for immunosuppressive effects on in vitro proliferative responses of activated $T$ cells. This paper also describes studies showing differences in CsA sensitivity of HLA-specific alloreactive T-cell clones generated from the MLR cultures and T-cell lines propagated from transplant biopsies.

\section{MATERIALS AND METHODS}

\section{Isolation and Separation of Cyclosporine $\mathbf{A}$ Metabolites from Human Bile}

Metabolites of CsA were obtained according to previously described methods [11]. Briefly, bile was collected from the T-tubes of adult patients who had undergone orthotopic liver transplantation and were receiving CsA therapy. A fresh $50-\mathrm{ml}$ bile aliquot was extracted with ethyl ether in a separatory funnel, and the ether was removed by evaporation under reduced pressure. The residue was taken up in aqueous methanol, washed twice with hexane, and evaporated to dryness. Multiple portions of the extract were injected onto an HPLC column (octadecylsilane, $5 \mu \mathrm{m}, 15 \mathrm{~cm}$ ) maintained at $70^{\circ} \mathrm{C}$. The mobile phase was a gradient of acetonitrile: water that was varied over $118 \mathrm{~min}$ to provide the optimal separation of the component peaks. The column eluent was monitored by ultraviolet detection at $214 \mathrm{~nm}$. Each of the major fractions was collected as it eluted from the column, was taken to dryness, and was combined with identical fractions from other HPLC injections. Purified samples of metabolites M1, M17, M18, and M21, kindly provided by G. Maurer (Sandoz, Basel, Switzerland) were used as standards to verify identical fractions of the eluate.

Thirteen major fractions were collected (see Figure 2). Fractions 8, 9, 10, and

FIGURE 2 Chromatogram of an HPLC injection of a bile extract. Absorbance at 214 $\mathrm{nm}$ over $118 \mathrm{~min}$ is shown, with the identification of each of the 13 fractions collected. Peaks with identical retention times to M17, M1, M18, and M21 are identified. The CsA was eluted after fraction 13 , and the peak is not shown in this figure.

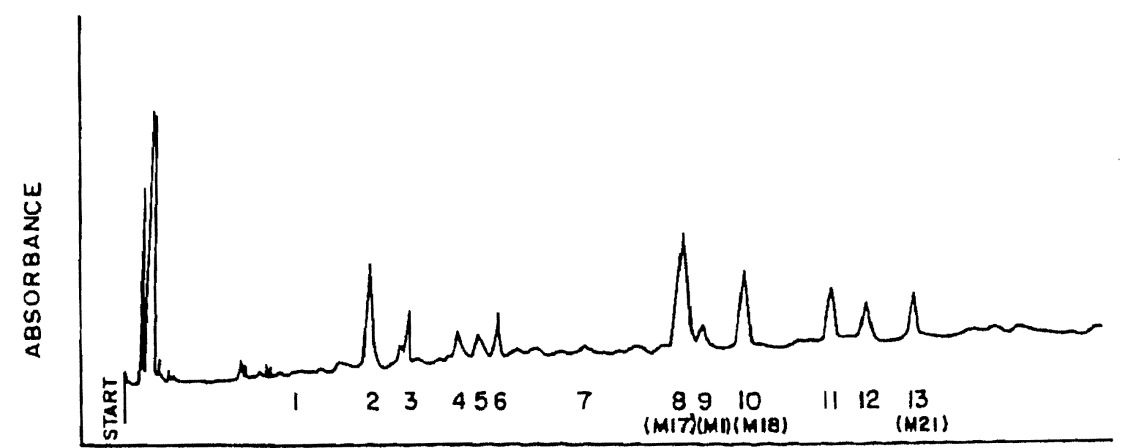

FRACTION NUMBER 
13 had identical retention times with the purified $M 17, M 1, M 18$, and $M 21$, and this was further confirmed by mass spectral analysis. Further HPLC analysis demonstrated that the bile-derived $\mathrm{M} 1, \mathrm{M} 18$, and $\mathrm{M} 21$ fractions were $>95 \%$ pure, whereas the bile-derived M17 fraction contained approximately $10 \%$ of unidentified material as determined from peak area.

Control bile was collected from pretransplant cirrhotic patients. The separation procedure was identical to the above protocol of separation of bile obtained from patients on CsA treatment. Fractions obtained from control bile were tested for their effect on lymphocyte proliferation.

\section{Lymphocyte Proliferation Assays}

Con A-induced activation. Human PBL were isolated from heparinized blood by Ficoll-Hypaque density gradient centrifugation. Using 96-well round-bottomed microplates, these cells $\left(10^{5} /\right.$ well) were incubated at $37^{\circ} \mathrm{C}$ in $200 \mu \mathrm{l}$ of tissue culture medium (TCM) supplemented with $10 \%$ human serum in the presence of Con $A(10 \mu \mathrm{g} / \mathrm{ml})$ for $72 \mathrm{~h}$. During the final $20 \mathrm{~h}$ of incubation, each culture was labeled with $1 \mu \mathrm{Ci}$ of $\left[{ }^{3} \mathrm{H}\right]$ thymidine. The cultures were harvested and counted in liquid scintillation counter as previously described [12].

Mixed lymphocyte culture-induced activation. Unidirectional human MLR cultures were set up with $10^{5}$ responder and $10^{5}$ irradiated (2000 R) stimulator cells in a volume of $200 \mu \mathrm{l} \mathrm{TCM}$ supplemented with $10 \%$ human serum for 6 days. Proliferation was assessed by the degree of $\left[{ }^{3} \mathrm{H}\right]$ thymidine incorporation during the final $20 \mathrm{~h}$ of incubation [12].

Primed lymphocyte test testing of alloreactive $T$ cells. Alloreactive human T-cell clones were generated from in vitro MLR cultures as previously described [12]. Alloreactive lymphocyte cultures were also propagated from human heart and liver core biopsies in the presence of IL-2 as previously reported [2,3]. Secondary proliferation of alloreactive cells was assessed in a 3-day primed lymphocyte test (PLT), whereby $10^{4}$ responder cells were incubated with $10^{5}$ irradiated $(2000$ R) stimulator cells [12]. Lymphocyte proliferation was assessed by $\left[{ }^{3} \mathrm{H}\right]$ thymidine incorporation as described above.

\section{Dose Effects of Cyclosporine A and its Metabolites} on Iymphocyte Proliferation

The inhibitory effects of CsA and its 13 bile-derived metabolite fractions on Con $A$ and MLR reactivity and PLT response of alloreactive lymphocytes were measured at different concentrations of drugs ranging from .005 to $5 \mu \mathrm{g} / \mathrm{ml}$. The results were expressed as a percentage inhibition using the formula:

$$
\text { Percentage inhibition }=\left(1-\frac{\mathrm{cpm} \text { with drug }}{\mathrm{cpm} \text { without drug }}\right) \times 100 \text {. }
$$

\section{RESULTS}

\section{Dose Effect of Cyclosporine A on Secondary Proliferation of Alloreactive T-Cell Clones}

Four class II and two class I HLA specific alloreactive T-cell clones were restimulated by the original MLR stimulator in the presence of different doses of CsA (Table 1). Three clones HAG, AR43 (both DRw6 specific), and AR62 (anti-B5?) 
TABLE 1 Dose effect of CsA on PLT responsiveness of alloreactive T-cell clones

\begin{tabular}{|c|c|c|c|c|c|c|c|c|c|c|}
\hline \multirow[b]{2}{*}{ Clone } & \multirow[b]{2}{*}{ Spec. } & \multirow{2}{*}{$\begin{array}{l}\text { Back- } \\
\text { ground } \\
\text { (cpm) }\end{array}$} & \multirow{2}{*}{$\begin{array}{l}\mathrm{PLT} \pm \mathrm{SD} \\
\quad(\mathrm{cpm})\end{array}$} & \multicolumn{7}{|c|}{$\begin{array}{l}\text { Percentage inhibition of PLT response } \\
\text { (Cyclosporine concentration, } \mathrm{ng} / \mathrm{ml} \text { ) }\end{array}$} \\
\hline & & & & $(500)$ & $(250)$ & (50) & (25) & (5) & $(0.5)$ & $(0.05)$ \\
\hline HAG & DRw6 & 320 & $51,608 \pm 1,887$ & 100 & 100 & 97 & 93 & 48 & 26 & 12 \\
\hline AR43 & DRw6 & 540 & $12,982 \pm 1,245$ & 98 & 98 & 96 & 91 & 50 & 18 & 0 \\
\hline AR62 & B57 & 379 & $37,718 \pm 2,789$ & 100 & 100 & 99 & 98 & 52 & 3 & 4 \\
\hline AR38 & A2 & 289 & $9,132 \pm 2,126$ & 97 & 99 & 70 & 57 & 30 & 0 & 2 \\
\hline AR 17 & DRw6 & 480 & $96,841 \pm 3,675$ & 100 & 100 & 62 & 47 & 21 & 10 & 10 \\
\hline DB29 & DQw 1 & 450 & $79,728 \pm 7,604$ & 100 & 97 & 54 & 43 & 22 & 16 & 13 \\
\hline
\end{tabular}

a The $10^{4}$ cloned cells were incubated with $10^{5}$ irradiated original stimulator cells for 3 days. Results represent the mean of triplicate wells.

${ }^{6} \mathrm{SD}$ is less than $15 \%$.

showed the highest CsA sensitivity. A concentration of $25 \mathrm{ng} / \mathrm{ml}$ was sufficient to induce greater than $90 \%$ inhibition. Three clones AR38 (anti-A2), AR17 (anti$D R w 6$ ), and DB29 (anti-DQw1 associated) were more resistant to the inhibitory effects of CsA: $250 \mathrm{ng} / \mathrm{ml}$ was needed to obtain more than $90 \%$ inhibition. These results show that alloreactive clones have different CsA sensitivity of their secondary proliferation. In this small group of T-cell clones, no differences in CsA sensitivity were shown to correlate with the specificity toward class I and II HLA antigens.

Cyclosporine A Effect on Donor-Specific Secondary Proliferation of Alloreactive Lymphocytes Propagated from Heart Transplant Biopsies

Nine heart biopsy-grown lymphocyte cultures were tested for donor-specific proliferative responses in the presence of various concentrations of CsA (Table 2). These biopsies were originally obtained from different patients at post-transplant intervals ranging from 15 to 162 days. Three cultures $\mathrm{HB} 5.83, \mathrm{HB} 12.87$, and HB 8.48 showed the highest CsA sensitivity. Four cultures required approximately tenfold higher amounts of CsA to reach the same degree of inhibition. HB 9.41 and $\mathrm{HB} 10.162$ showed a considerable resistance to the in vitro immunosuppressive effects of CsA. Even at a highest tested concentration of 50 $\mathrm{ng} / \mathrm{ml}$, there was only slightly above $50 \%$ inhibition of donor-specific proliferation. These lymphocytes originated from heart transplant biopsies obtained from two patients following treatment of an acute rejection episode. It seems possible that the increased immunosuppression used to treat the rejection episode had a selective effect on graft-infiltrating lymphocytes, whereby only the more resistant cells remained and subsequently could be propagated from the biopsy.

These observations demonstrate differences in CsA sensitivity of lymphocyte cultures propagated from heart transplant biopsies, and these findings are consistent with differences in CsA sensitivity of alloreactive T-cell clones.

Inhibition of Con A-Induced Proliferation by Bile-Derived Cyclosporine A Metabolite Fractions

The CsA and 13 HPLC fractions of bile-derived CsA metabolites were tested for their inhibitory effect on Con A-induced proliferation of PBL (Figure 3). Greater 
TABLE 2 Cyclosporine A effect on the donor-specific PLT response of lymphocyte cultures generated from transplant heart biopsies

\begin{tabular}{|c|c|c|c|c|c|c|}
\hline \multirow[b]{2}{*}{ HB culture } & \multirow{2}{*}{$\begin{array}{l}\text { Background } \\
\text { (cpm) }\end{array}$} & \multirow{2}{*}{$\begin{array}{c}\text { PLT } \\
\text { Donor } \pm \mathrm{SD}^{6} \\
(\text { cpm })\end{array}$} & \multicolumn{4}{|c|}{$\begin{array}{c}\text { Percentage inhibition of } \\
\text { PLT response } \\
\text { (Cyclosporine concentration, } \mathrm{ng} / \mathrm{ml} \text { ) }\end{array}$} \\
\hline & & & $(50)$ & (5) & $(0.5)$ & $(0.05)$ \\
\hline 5.83 & 12,992 & $75,462 \pm 3,773$ & 99 & 99 & 65 & 35 \\
\hline 12.87 & 667 & $30,431 \pm 1,825$ & 99 & 99 & 48 & 20 \\
\hline 8.48 & 768 & $12,474 \pm 997$ & 97 & 73 & 26 & 18 \\
\hline 10.142 & 356 & $11,028 \pm 1,016$ & 94 & 58 & 36 & 5 \\
\hline 7.147 & 350 & $54,628 \pm 4,516$ & 98 & 58 & 13 & 10 \\
\hline 4.39 & 480 & $27,504 \pm 1,925$ & 90 & 52 & 5 & 2 \\
\hline 6.15 & 207 & $22,498 \pm 1,034$ & 93 & 35 & 1 & 0 \\
\hline 9.41 & 368 & $7,158 \pm 572$ & 58 & 12 & 3 & 0 \\
\hline 10.162 & 341 & $34,547 \pm 3,145$ & 54 & 11 & 0 & 0 \\
\hline
\end{tabular}

- Each heart biopsy (HB) culture is referred to by two numbers. The first number identifies the patient and the second number refers to the day post transplant when the biopsy was obtained.

' Results represent the mean of triplicate wells.

'SD is less than $15 \%$.

than $80 \%$ inhibition was observed with CsA doses at concentrations of $.5 \mu \mathrm{g} / \mathrm{ml}$ or greater. Several fractions, especially $8,9,10$, and 13 , induced significant inhibition of Con A-induced proliferation, but higher concentrations were necessary, namely $5 \mu \mathrm{g} / \mathrm{ml}$. No effect was noted for any of these fractions at $.05 \mu \mathrm{g} / \mathrm{ml}$ or lower. The highest degree of inhibition (about $50 \%$ ) was observed with fractions 8 and 9, which primarily contained metabolites $\mathrm{M} 17$ and $\mathrm{M} 1$, respectively. Both fractions required about 100-fold higher concentrations than the $.05 \mu \mathrm{g} / \mathrm{ml}$ concentration of CsA to induce a $50 \%$ inhibition of the Con A response. Other fractions including 10 and 13 , which contained metabolites M18 and M21,

FIGURE 3 Inhibition of Con A-induced proliferation by CsA and 13 bile-derived metabolites. (Left) Dose effect of $C s A$ and metabolites $M 17, M 1$, and $M 21$. (Right) Effect of bile-derived CsA metabolite fractions used at concentration of $5 \mu \mathrm{g} / \mathrm{ml}$. Fractions corresponding to M17, M1, M18, M21, M13, M9, and M8 are identified. Representative results of duplicate experiments. Con A response was $71,624 \pm 2,704 \mathrm{cpm}$.
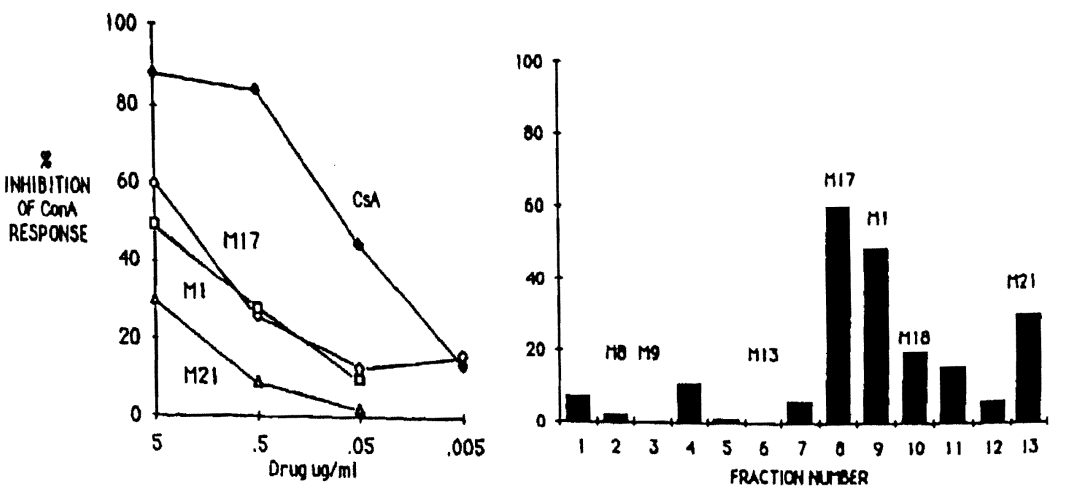

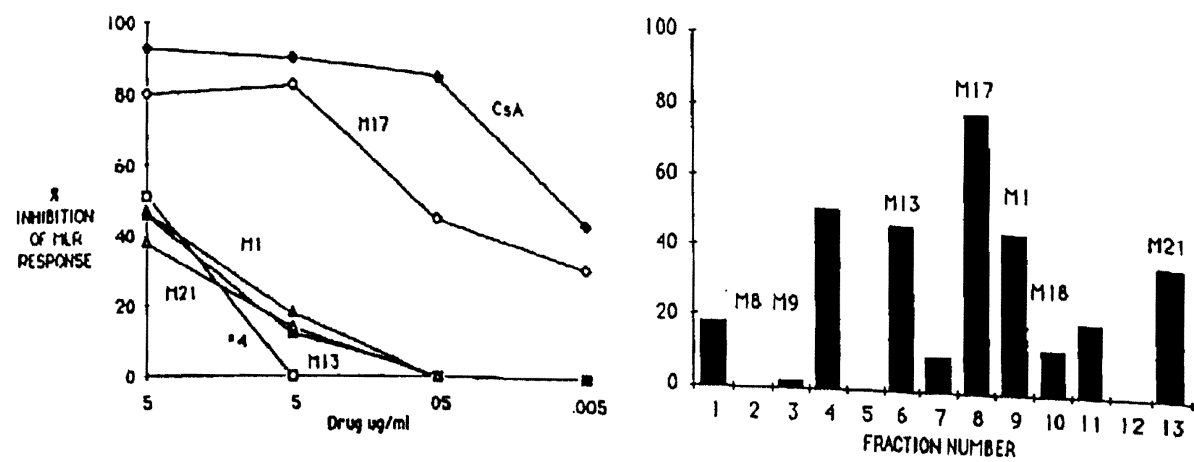

FIGURE 4 Effect of CsA and its metabolites on MIR-induced proliferation. (Left) Dose effect of CsA and metabolites M17, M1, M21, M13, and fraction 4. (Right) Inhibition of MLR response in the presence of $5 \mu \mathrm{g} / \mathrm{ml}$ of CsA metabolite fractions. Representative results of duplicate experiments. MLR response was $23,168 \pm 1,335 \mathrm{cpm}$.

showed even weaker inhibitory effects on Con A-induced proliferation. These studies demonstrate that HPLC fractions of bile-derived CsA metabolites had considerably lower inhibitory effects on Con $A$ responsiveness of PBL.

Mixed Leukocyte Reaction Inhibition by Bile-Derived

Cyclosporine A Metabolites

The HPLC fractions were studied for their effect on the primary MLR (Figure 4). Greater than $80 \%$ MLR inhibition was observed for CsA at doses as low as 0.05 $\mu \mathrm{g} / \mathrm{ml}$. Significant inhibition was observed with fractions $4,6,8,9$, and 13 at 5 $\mu \mathrm{g} / \mathrm{ml}$ concentrations. Especially, fraction 8, which contained M17, exhibited comparable inhibitory activity observed with $5 \mu \mathrm{g} / \mathrm{ml}$ of CsA. Even a lower concentration of fraction 8 at $0.5 \mu \mathrm{g} / \mathrm{ml}$, there was almost $80 \%$ inhibition. On the other hand, no significant inhibitory effect was observed for the other fractions at concentrations of $0.5 \mu \mathrm{g} / \mathrm{ml}$.

These observations demonstrate that several HPLC-fractionated CsA metabolites in bile have significant inhibitory effects on MLR-induced proliferation. Especially fraction 8 (M17) was strongly inhibitory, almost approaching that of CsA itself. This is in contrast to the considerably lower inhibitory effects of CsA metabolites on Con A-induced proliferation.

Fractions obtained from control bile showed no inhibitory activity on lymphocyte proliferation induced by Con A or MLR (data not shown). These results indicate that the HPLC-fractionated CsA metabolites found in bile are not nonspecifically toxic to $\mathrm{T}$ lymphocytes.

Cyclosporine A Metabolite Effects on Primed Lymphocyte Test Responses of Alloreactive T.Cell Clones

Two alloreactive T-cell clones HA-G (anti-DRw6) and DB29 (anti-DQw1 associated) were tested in secondary proliferation assays in the presence of bile metabolites of CsA fractionated by HPLC (Figures 5 and 6 ). As may be seen in Table 1 , clone $\mathrm{HAG}$ was more sensitive to CsA than clone DB29. The strongest inhibitory effects were observed with fractions 8,9 , and 13 . The differences in CsA sensitivity berween clunes $\mathrm{HAG}$ and DB29 were also observed for the metabolites. At a concentration of $5 \mu \mathrm{g} / \mathrm{ml}$, fraction 13 , which contained metabolite M21, was 

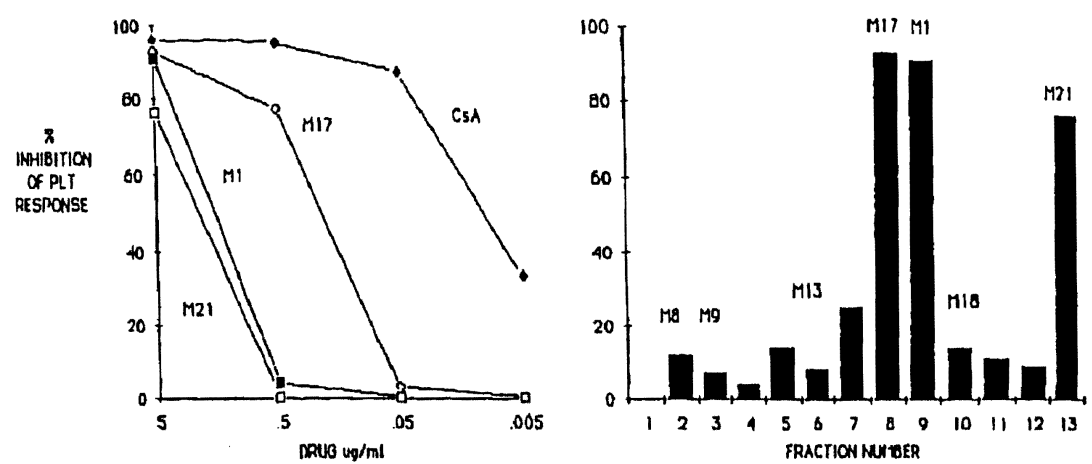

FIGURE 5 Inhibition of PLT reactivity of clone HAG by CsA metabolite fractions. (Left) Dose effect of CsA and metabolites M17, M1, and M21. (Right) Effect of CsA metabolite fractions $(5 \mu \mathrm{g} / \mathrm{ml})$ on the PLT response. Representative results of triplicate experiments. PLT response of HAG was $12,069 \pm 1,301 \mathrm{cpm}$.

strongly inhibitory to CsA-sensitive HA6 but not to DB29. Also, the inhibition by fraction 9 , which contained M1, was higher for HA6 than for DB29. Figure 5 shows that fraction 8 , which contained $M 17$, had equally high inhibitory effects on HAG and DB29. It may also be seen that several fractions showed weak inhibitory effects on HA6 but not DB29. These findings show that similar differences in CsA sensitivity among alloreactive T-cell clones may also be observed for the various CsA metabolites.

\section{Cyclosporine A Metabolite Effects on Liver Transplant \\ Biopsy-Grown Lymphocytes}

The CsA and four bile-derived metabolites (M17, M1, M18, and M21) were tested for their inhibitory effect on donor-specific PLT response of alloreactive lymphocytes generated from a liver transplant biopsy. As shown in Table 3, all

FIGURE 6 Inhibition of PIT response of alloreactive clone DB29. (Left) Dose effect of CsA and metabolites M17, M1, and M21. (Right) Inhibition profile of PLT response in the presence of CsA metabolite fractions $(5 \mu \mathrm{g} / \mathrm{ml})$. Representative results of triplicate experiments. PLT response of DB29 was $25,377 \pm 1,376 \mathrm{cpm}$.
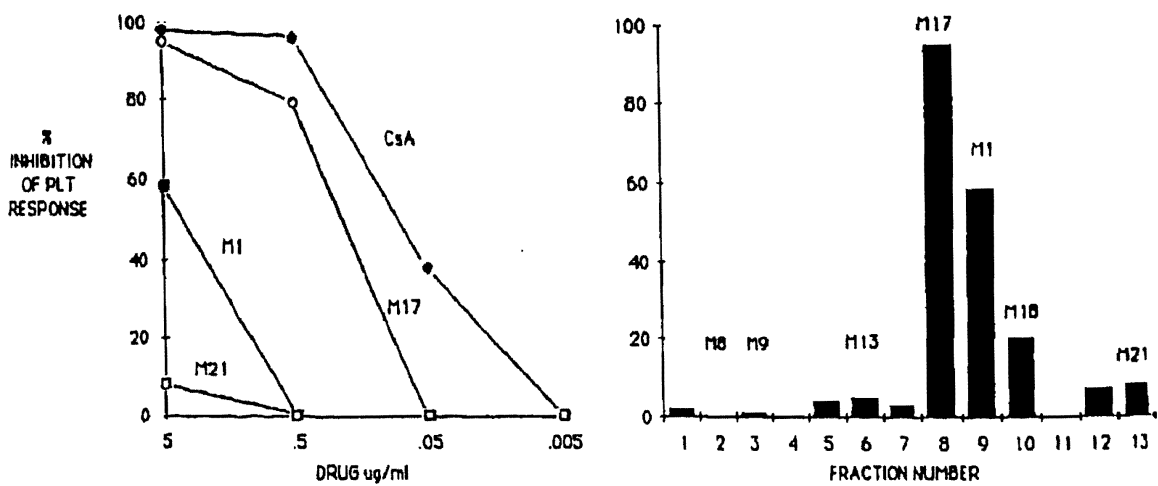
TABLE 3 Dose effect of CsA and metabolites M17, M1, M18, and M21 on PLT response of lymphocyte cultures generated from transplant liver biopsy

\begin{tabular}{lccccc}
\hline \multirow{2}{*}{$\begin{array}{c}\text { Drug } \\
\text { concentration } \\
(\mu \mathrm{g} / \mathrm{ml})\end{array}$} & \multicolumn{5}{c}{ Percentage inhibition of PLT response } \\
\cline { 2 - 6 } & CsA & M17 & M1 & M18 & M21 \\
\hline 5.0 & 99 & 99 & 99 & 85 & 99 \\
0.5 & 98 & 94 & 91 & 52 & 69 \\
0.25 & 96 & 84 & 73 & 28 & 50 \\
0.05 & 94 & 27 & 25 & 30 & 25 \\
0.005 & 40 & 7 & 13 & 40 & 24 \\
\hline
\end{tabular}

- The PLT response of liver biopsy alloreactive lymphocytes against donor cells was $20,330 \pm 1,568$ cpm. Results represent mean of duplicate cells. SD is less than $15 \%$.

four CsA metabolites tested strongly inhibited the PLT reactivity of liver biopsy lymphocytes at $5 \mu \mathrm{g} / \mathrm{ml}$. In addition, metabolites M17 and M1 exhibited comparable inhibitory activity observed with all doses of parent compound CsA. These results indicate that bile-derived CsA metabolites have significant inhibitory effects on alloreactive $T$ cells derived from cellular infiltrates of liver allograft.

\section{DISCUSSION}

This study was designed to assess the in vitro immunosuppressive effects of various CsA metabolites in human bile from liver transplant patients. Besides Con $A$ responsiveness and primary MLR activation of PBL, the assays focused on the secondary proliferative responses of cloned alloreactive $T$ cells and transplant biopsy-grown lymphocytes. These studies also considered differences between activated $T$ cells in their sensitivity to CsA and its metabolites. This seemed particularly relevant to allograft infiltrating lymphocytes, which, in the allogeneic environment of the graft may undergo further proliferation and subsequently cause transplant rejection. The intragraft levels of $\mathrm{CsA}$ and its metabolites would likely contribute to the outcome of the transplant.

Secondary proliferation experiments on MLR-generated alloreactive T-cell clones showed significant differences in CsA responsiveness among the various clones. Certain clones required tenfold larger doses than other more "sensitive" clones. These observations are similar to the findings by Manca et al [6], who studied MLR-derived T-cell lines. Our data indicate that CsA sensitivity appears independent from the specificity of alloreactive T-cell clones toward class I and II HLA antigens.

Differences in CsA sensitivity were also observed with lymphocyte cultures propagated from heart transplant biopsies. These activated $T$ cells often exhibit allospecificity toward donor HLA antigens $[2,4,13]$. Although the presence of alloreactive $T$ cells correlated with allograft rejection, on many occasions there is little evidence of an ongoing or upcoming rejection episode. The latter might to due to suppressor cells that inhibit cell-mediated effector mechanisms leading to rejection. Another possibility that the CsA sensitivity of graft infllating on nine factor whether or not the allograft undergoes rejection. In tange of differences biopsy-grown lymphocyte cultures, we observed a 100 -fold tively $\mathrm{CsA}$-insensitive in CsA sensitivity. Of particular interest were the two relatively treatment of a cultures that were grown from biopsies after a steroid bolus treatment of 
rejection episode. Such treatment results in a marked depletion of lymphocytes in the cellular infiltrates, and it seems that a subset of CsA-insensitive lymphocytes was still present after the steroid treatment. More studies are needed to establish a correlation between CsA sensitivity and transplant outcome.

The effectiveness of CsA therapy must consider the immunosuppressive effects of individual metabolites. The presence of MLC-inhibitory activity in the serum of renal transplant recipients when low levels of parent drug were found suggested that CsA metabolites may contribute to the immunosuppressive activity [14]. Freed et al [10] have demonstrated that $M 17, M 1$, and $M 21$ are the most active $\mathrm{CsA}$ metabolites as assessed by in vitro inhibition of mitogen responses, allogeneic proliferation, and the inhibition of IL-2 production during MLC. Our studies have also showed that bile fractions containing $\mathrm{M} 17$ followed by $\mathrm{Ml}$ and M21 have most significant in vitro immunosuppressive effects. This is particularly apparent in Con $A$ and primary MLR stimulation assays, in which Cs $A$ was found to be about 100-fold more inhibitory than M17, the most active metabolite. On the other hand, only 10-fold differences between $\mathrm{Cs} A$ and the most active metabolites were observed in the inhibition of the secondary proliferation of alloactivated $\mathrm{T}$ cells. This trend was present with alloreactive $\mathrm{T}$-cell clones and liver transplant biopsy-propagated cell cultures. Thus, it seems that CsA metabolites may have relatively greater inhibitory effects on alloreactive $T$ cells, including those found in cellular infiltrates of allografts, than on the primary activation of $\mathrm{T}$ cells.

The exact contribution of $\mathrm{CsA}$ metabolites to the overall immunosuppressive activity depends on the relative concentrations of these metabolites in transplant patients. It appears that M17 is a major metabolite of $\mathrm{CsA}$ and is present in concentrations greater than that of CsA in kidney [9], liver, and heart transplant patients [15]. A comprehensive therapeutic strategy should then include monitoring of not only CsA but also the active metabolites.

\section{ACKNOWLEDGMENTS}

This work was supported by NIH Grants AI 23467, HL 36416, and AM 34475 and by Sandoz Pharmaceuticals, East Hanover, New Jersey. The authors would like to thank the Pittsburgh Transplant Foundation for their aid in donor sample procurement. We also would like to thank Drs. Bartley Griffith, Robert Hardesty, and Robert Kormos for their cooperation. The expert technical help of Loretta DiVecchia was greatly appreciated.

\section{REFERENCES}

1. Mayer T, Fuller A, Fuller T, Lazarovits A, Boyle L, Kurnick J: Characterization of in vivo activated allospecific $T$ lymphocytes propagated from human renal allograft biopsies undergoing rejection. J Immunol 134:258, 1985.

2. Zeevi A, Fung J, Zerbe T, Kaufman C, Rabin B, Griffith B, Hardesty R, Duquesnoy $R$ : Allospecificity of activated $T$ cells grown from endomyocardial biopsies from heart transplant patients. Transplantation 41:620, 1986.

3. Fung J, Zeevi A, Starzl T, Demetris A, Iwatsuki S, Duquesnoy R: Functional characterization of infiltrating $\mathrm{T}$ lymphocytes in human hepatic allografts. Hum Immunol 16:182, 1986.

4. Weber T, Kaufman C, Zeevi A, Zerbe TR, Hardesty RJ, Kormos RH, Griffith BP, Duquesnoy RJ: Propagation of lymphocytes from human heart transplant biopsies: Methodological aspects. Transplant Proc, in press, 1987. 
5. Sjoberg O, Totterman TH: Variations in inhibition by cyclosporine A of the proliferative response, using different individuals and $T$ cell stimulators. Transplant Proc 18:40, 1986.

6. Manca F, Carozzi S, Kunk I A, Barocci S, Dessi V, Valente V, Fontana I, and Celada F: In vitro sensitivity to cyclosporine is clonally distributed among alloreactive clones. Transplant Proc 17:71, 1985.

7. Kahan BD, Ried M, Newburger J: Pharmacokinetics of Cyclosporine in human renal transplantation. Transplant Proc 15:466, 1983.

8. Venkataramanan R, Starzl TE, Yang S, Burckart GJ, Ptachcinski RJ, Shaw BW, Iwatsuki S, Van Thiel DH, Sanghvi A, Seltman H: Biliary excretion of cyclosporine in liver transplant patients. Transplant Proc 18:46, 1986.

9. Rosano TG, Freed BM, Cerilli J, Lempert N: Immunosuppressive metabolites of cyclosporine in the blood of renal allograft recipients. Transplantation 42:262, 1986.

10. Freed $B$, Rosano TG, Maurer $G$, Lempert $N$ : In vitro immunosuppressive properties of cyclosporine metabolites. Transplantation 43:123, 1987.

11. Burckart GJ, Starzl TE, Yenkataramanan R, Hashim H, Wang L, Wang P, Makowka L, Zeevi A, Ptachcinski RJ, Knapp JE, Iwatsuki S, Esquivel C, Sanghvi A, Van Thiel $\mathrm{DH}$ : Excretion of cyclosporine and its metabolites in human bile. Transplant Proc $18: 46,1986$.

12. Zeevi A, Duquesnoy $R$ : Specificity of alloactivated human $T$ lymphocyte clones in secondary proliferation, cell mediated lympholysis and interleukin 2 release. J Immunogenet $12: 17,1985$.

13. Fung J, Zeevi A, Markus B, Zerbe T, Duquesnoy RJ: Dynamics of allospecific T lymphocyce infiltration in vascularized human allografts. Immunol Res 5:149, 1986.

14. Kahan BD, Van Buren CT, Lorber MI, Flechner SM, Wideman CA, Kerman RH: Optimization of cyclosporine immunosuppression for renal transplantation. Transplant Proc 12:35, 1985.

15. Wang CP, Burckart G, Ptachcinski R, Venkataramanan R, Schwinghammer $T$, Hakala T, Griffith B, Hardesty R, Shadduck R, Starzl T: Cyclosporine metabolite concentracions in blood in renal, liver, heart, and bone marrow transplant patients. Transplant Proc, in press, 1987. 